\title{
Neighbour-Cooperation Heterogeneity-Aware Traffic Engineering for Wireless Sensor Networks
}

\author{
Christopher Mumpe ${ }^{\star, 1}$, Da Tang ${ }^{\star, 2}$, Muhammad Asad ${ }^{\dagger, 3}$, Muhammad Aslam $^{\ddagger, 4}$, \\ Jing Chen ${ }^{\star}, 5$, Jinsi Zhu ${ }^{\star, 6}$, Luyuan Jin $^{\star, 7}$ \\ ${ }^{\star}$ School of Computer Science and Technology, Dalian University of Technology. Dalian - China \\ ${ }^{\dagger}$ Department of Computer Science and IT, Superior University, Gold Campus, Lahore, Pakistan \\ ${ }^{\ddagger}$ Department of Computer Science, COMSATS University Islamabad, WAH CANTT, Pakistan
}

\begin{abstract}
Extending the operational duration is a major field of interest in Wireless Sensor Networks (WSNs). This lifetime enhancement task challenges researchers to design an energy efficient traffic engineering which minimizes the dissipation energy and retain the expected quality of routing protocols. Network lifetime can be prolonged by balancing the energy optimization throughout the network period over which sensors relay data traffic towards Base Station (BS). Existing techniques of continuous and autonomous reporting sensor nodes, offer an opportunity to design the sensing and reporting co-operation between sensor nodes. Nearby nodes with similar reading environment can cooperate with each other to avoid transmission redundant information. In this paper we propose "Adaptive Inter-Networking Improved (AINI)" multi-hop routing protocol with co-operate sensing of inter and intra cluster communication by exploiting the concept of tripling the sensor nodes. Proposed routing protocol improved the reliability of whole network by improving the reliability of inter-cluster multi-hoping. Sensor nodes use the shortest path to deliver data to $\mathrm{CH}$ using intra-cluster multihoping and these CHs are accountable to forward this data to BS using inter-cluster multi-hop communication. Proposed routing protocol resolves the certain issues of WSN like network lifetime, network stability and $\mathrm{CHs}$ selection technique. To prove the efficiency of our proposed model we compared the simulation results with existing state-of-the art routing protocols such as, LEACH, LEACH-C, SEP, ESEP and DEEC. Experimental results shows the benefits of neighbour cooperation and heterogeneityaware by the performance of proposed protocol over existing state-of-the-art routing protocols.
\end{abstract}

Keywords-Wireless Sensor Networks; energy efficient; clustering; multi-hop; routing protocol

\section{INTRODUCTION}

Wireless Sensor Network (WSN) comprises a large number of sensor nodes which used to measure and monitor the field such as health monitoring, battlefield surveillance, sensing of light, sound, traffic monitoring, industrial control, vibration, humidity, temperature, etc [1]. After sensing, sensor nodes forward this information to the Base Station (BS). Sensor nodes are randomly deployed in hostile environments which are equipped with limited battery power and processing capabilities with the objective of data collection from sensing field and deliver it to user interface for analysis [2], [3]. Energy efficient routing is a major issue to resolve in WSNs as efficient routing helps to prolong the connectivity of sensor nodes with the network [4]. Stability and lifetime enhancements for WSNs without negotiating the requirement of network are the main aim of on-going research. In order to achieve these aims of WSNs, the network can be divided into groups called clusters. Dynamic CH selection was considered to select a suitable $\mathrm{CH}$ which can be implemented with a centralized or a distributed approach in WSNs [5]. Whereas, heterogeneous routing protocol comes under the hierarchy of centralized and distributed clustering [6]. It is noticed that clustering can also improve the load balancing, scalability and connectivity of the network [7], [8]. Certain Cluster Head (CH) selection criterion is used to select a $\mathrm{CH}$ for a specific cluster. This $\mathrm{CH}$ is responsible for special tasks to perform for its specific cluster such as, assigning TDMA slots to sensor nodes for receiving data, compress this data with complex calculation and transmit it to BS. In this way, energy of $\mathrm{CH}$ dissipates much earlier than other sensor nodes which leads to the uneven energy dissipation among the network [9]. For this purpose, CHs should be elected dynamically in each round for load balancing and maintain the energy level of network [10]. Various routing protocol have been proposed using distributed clustering [11], these clustering routing protocols generate random $\mathrm{CH}$ selection based on probabilistic method, which usually results into un-even cluster formation [12]. This distributed clustering choose $\mathrm{CHs}$ randomly, by ignoring the residual energy which leads to earlier expiration of low energy nodes [13]. Thus, uniform distributed clustering is expected to resolve this uneven energy dissipation of $\mathrm{CHs}$ by allocating the variable to highest energy level nodes inside particular cluster [14], [15]. Figure 1 shows the example of distributed clustering.

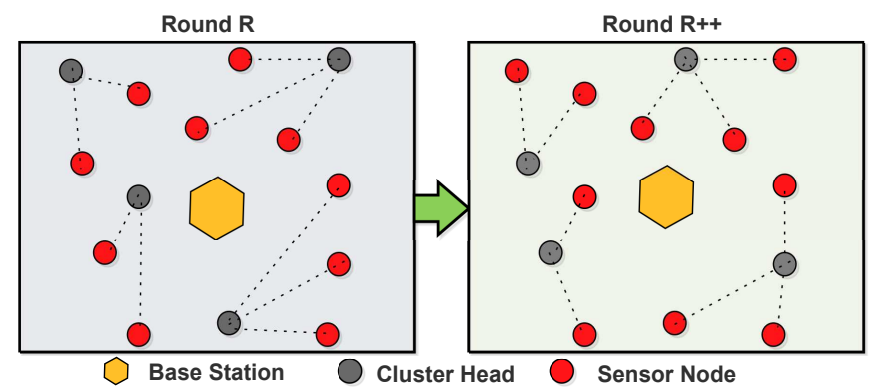

Fig. 1. Sample of Distributed Clustering

In order to sense data in complex environment heterogeneous sensor nodes are used to enhance the nodes capability and functionality [16]. For example, sensor nodes sparsely deploy at higher hierarchy layers are expected to execute more complex tasks. In this paper an heterogeneous clustering routing protocol named "AINI: Adaptive Inter-Networking Improved" routing protocol for WSNs is proposed. To improve the lifetime and stability of network (intra-cluster for nodes to $\mathrm{CHs}$ and inter-cluster for $\mathrm{CHs}$ to $\mathrm{BS}$ ) multi-hop commu- 
nication is used. Existing dynamic state-of-the art clustering routing protocol like LEACH, LEACH-c, SEP, ESEP, and DEEC for WSNs have achieved better performance of network. Trailing their ideas, we propose a new concept of tripling the sensor nodes into Child Sensor Nodes (CSN) which has further improved the $\mathrm{CH}$ selection technique stability and lifetime of network.

The rest of the paper is organized as follows: Section 2 contains the brief literature review of existing work. Section 3 described the network model of proposed routing protocol. Proposed model, network operations and $\mathrm{CH}$ selection technique is briefly explained in Section 4. Section 5 defines the performance measures. Experimental results and simulations are discussed in Section 6. Experimental methodology is given in Section 7. Conclusion and future work are described in Section 8.

\section{RELATED WORK}

Extensive research on routing protocols has been proposed based on structure and hierarchy of the WSNs. Low Energy Adaptive Clustering Hierarchy (LEACH) [17], is a pioneer clustering routing protocol, many routing protocols were proposed based on it. In this literature review, we are going to mention few protocols from which our protocol is encouraged and was able to overcome the problems over previous routing protocols. LEACH divides the network into clusters and assigns a $\mathrm{CH}$ to each cluster for communication with BS. These $\mathrm{CHs}$ are randomly chosen and the rest of the nodes are called member nodes of cluster. These CHs have added responsibility to receive data from all nodes and transmit it to BS which causes $\mathrm{CHs}$ to dissipate more energy than member nodes and causes the early death. The problem in LEACH is, it is distributed and only designed for homogeneous network. In [18], LEACH-centralized is proposed to overcome the existing issue, though it improves the network lifetime over LEACH but due to the limited scalability LEACH-c could not perform well in large scale networks. In [19], Stable Election Protocol (SEP) two-level heterogeneous network is proposed. SEP introduces two types of nodes, nodes with higher energy are referred as advance nodes and the other nodes are called normal nodes. Most probability to become a $\mathrm{CH}$ is of advance nodes as compared to normal nodes. Problem in SEP was, it performs distributed cluster-formation which results the uneven number of clusters and secondly it only allows for two-level heterogeneity. In order to further enhance SEP protocol Enhanced-SEP [20] protocol was proposed with three level heterogeneity. In [21] Distributed Energy Efficient Clustering (DEEC) proposed heterogeneous network with distributed property. In DEEC, CHs are chosen according to the initial and residual energy of nodes; furthermore, DEEC holds multi-level heterogeneity but the problem with DEEC is, it generates uneven $\mathrm{CHs}$ in each round. In [22], Hierarchical Cost Effective LEACH (HCEL) protocol proposed three-level heterogeneous network model. This protocol intends to perform better in heterogeneous network in terms of network lifetime and stability. But the simulation results of HCEL shows gradual reduction of deployment cost ratio in dense network. In [23], Advanced-Multi-Hop LEACH proposed for heterogeneous network, which uses optimal path for communication between $\mathrm{CH}$ and $\mathrm{BS}$. The authors claim that using optimal number of clusters and hops using multi-hoping can results better network lifetime and stability period over singlehop LEACH. In [24], Threshold sensitive Energy Efficient sensor Network (TEEN) routing protocol proposed for reactive networks. It was simple temperature sensing protocol, major drawback of this protocol was that, it continuously sense the field and transmit only when there is a change in data values. To enhance the TEEN protocol, Distance Adaptive Threshold sensitive Energy Efficient sensor Network [25] (DAPTEEN) was proposed. DAPTEEN enhanced the network lifetime and $\mathrm{CH}$ selection technique of TEEN. In this literature review, various routing protocols are presented and each research contribution tries to overcome the energy dissipation problem and added some improvements in network lifetime of WSNs. Some protocols enhance the stability of network while some protocols added more heterogeneity in nodes. In this regard we propose AINI: Adaptive Inter-Networking Improved routing protocol, with advance $\mathrm{CH}$ selection and improved routing technique. Proposed protocol and its models are described in next sections.

\section{Network Model FOR Proposed Protocol}

Proposed routing protocol develops cluster-formation and then proceeds tripling connectivity among selected $\mathrm{CHs}$. In this way the proposed model allows general network model settings and shows enough flexibility of execution within any random deployment of sensor nodes. Proposed network model considers some key assumptions such as; (1) linear radio model of radio characteristics to carry on wireless communications, (2) static nodes deployment to ensure nodes maintain certain location-ID throughout the network operations, (3) contain heterogenous characteristics according to the energy resources during network operation and (4) development of multi-hop intra cluster communication and multi-hop inter cluster communications during transmission of sensed information. Our goal is to minimize the energy dissipation in order to improve the network stability and lifetime. Execution of proposed model create unique clustering in which nodes perform tripling bonding and then start communication with selected CHs. Network topology of proposed protocol is shown in Figure 2

\section{Proposed Model of AINI Protocol}

Our proposed model AINI routing protocol provides distributed cluster-formation of sensor nodes to enable dynamic self-organized management among sensor nodes based on probabilistic cluster-head selection. Proposed model AINI designs unique distributed cluster-formation in which initially sensor nodes select themselves as Parent Sensor Nodes (PSNs) and later on these PSNs develop unique collaborations among neighbour PSNs to form tripling bonding among every three closer PSNs. These tripling-based bonded PSNs are defined as Child Sensor Nodes (CSNs). Major purpose of formation of CSNs bonding is to introduce higher level assistance among CSNs which result into creation of single Leading Sensor Node (LSNs) which will transmit the information towards selected CHs on behalf of its bonded CSNs. This unique two layer distributed neighbour-formation of PSNs and CSNs offer reasonable energy efficiency by assistance of LSNs, which is almost three times more than some conventional distributed routing protocols. 


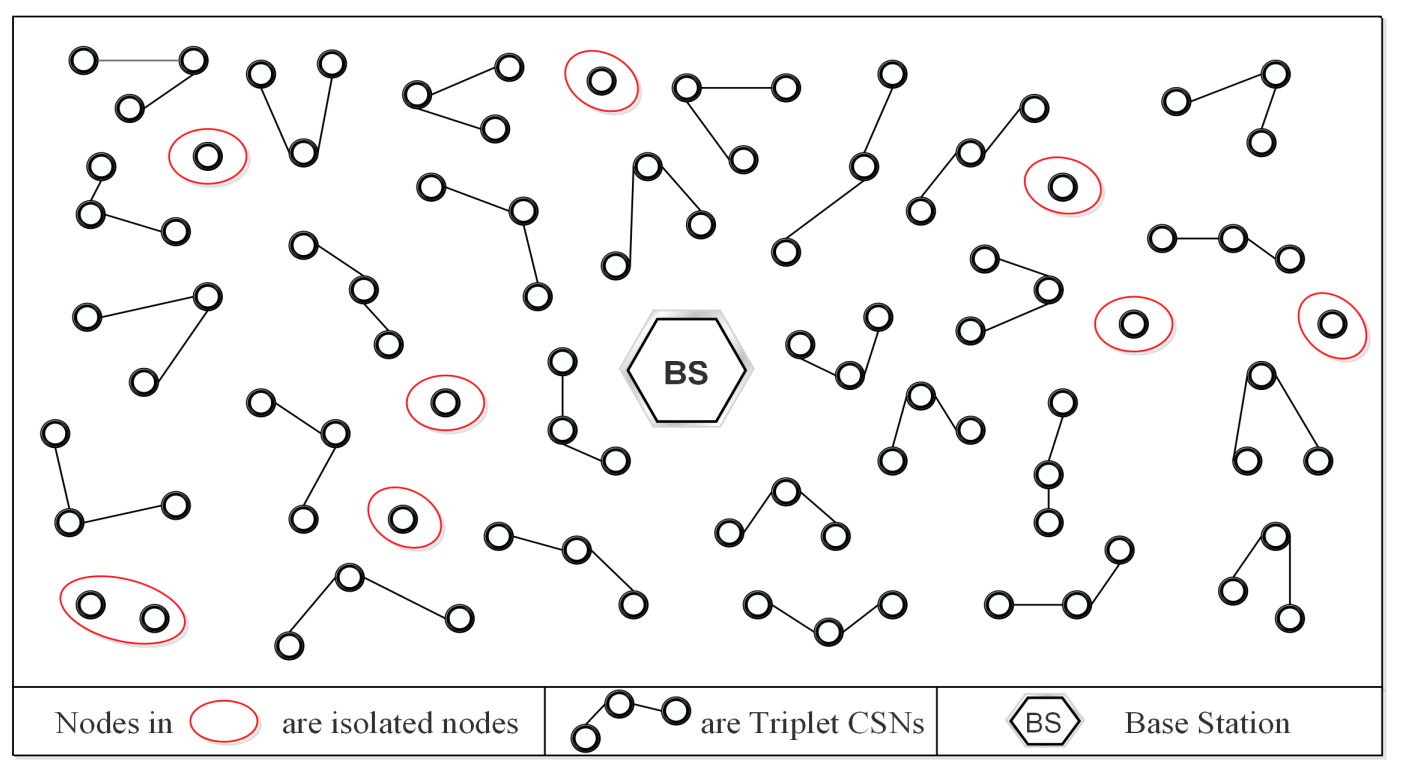

Fig. 2. Network Topology of AINI

During each round of network, all CSNs switch between 'Sleep' and 'active' modes. After forming of CSNs, one LSN will act as active node and becomes responsible for data transmissions towards $\mathrm{CHs}$. During this transmission period of active nodes, transceiver of other nodes will be in sleepmode, this sleep-mode will stop communication with their $\mathrm{CHs}$ and nodes only sense the environment. Only one node will be active in each CSNs for one round, other two nodes will be in sleep-mode. In this way, nodes will rotate the transmission responsibility and will serve after consecutive two rounds and will save its residual energy considerably. These two nodes in sleep-mode will save additional energy by avoiding idle listening. Nodes which are not in any CSNs will be active and communicate continuously with $\mathrm{CHs}$ in all rounds until their energy depleted. Networking operation of proposed protocol is divided into $R_{n}$ rounds and each round consists of four phases: Initialization phase, Network setup phase, Transmission phase and Termination phase.

AINI protocol is mainly proposed to generate an energy efficient distributed clustering routing protocol which improves highly demanded QoS, reliability, better network lifetime and network stability by minimizing the overall energy consumption. Furthermore, multi-hop routing minimizes the packet drop ratio and retransmission of packet. AINI enforces adaptive $\mathrm{CH}$ selection which is accountable to update nodesmode.Figure 3 shows the four major network operations in flowchart of proposed routing protocol. The main communication steps of AINI protocol are following:

\section{A. Cluster Head Selection}

Proposed routing protocol is completely distributed and nodes help themselves to become CHs. Selection process require iterations to run which should be long enough to receive message from the nodes in the range of cluster. Initially nodes are deployed in heterogeneous mode with different energy levels. As in [17], [18], [19], [20], [21], [24], [26], [25] every node is forced to become a CH because of rotating

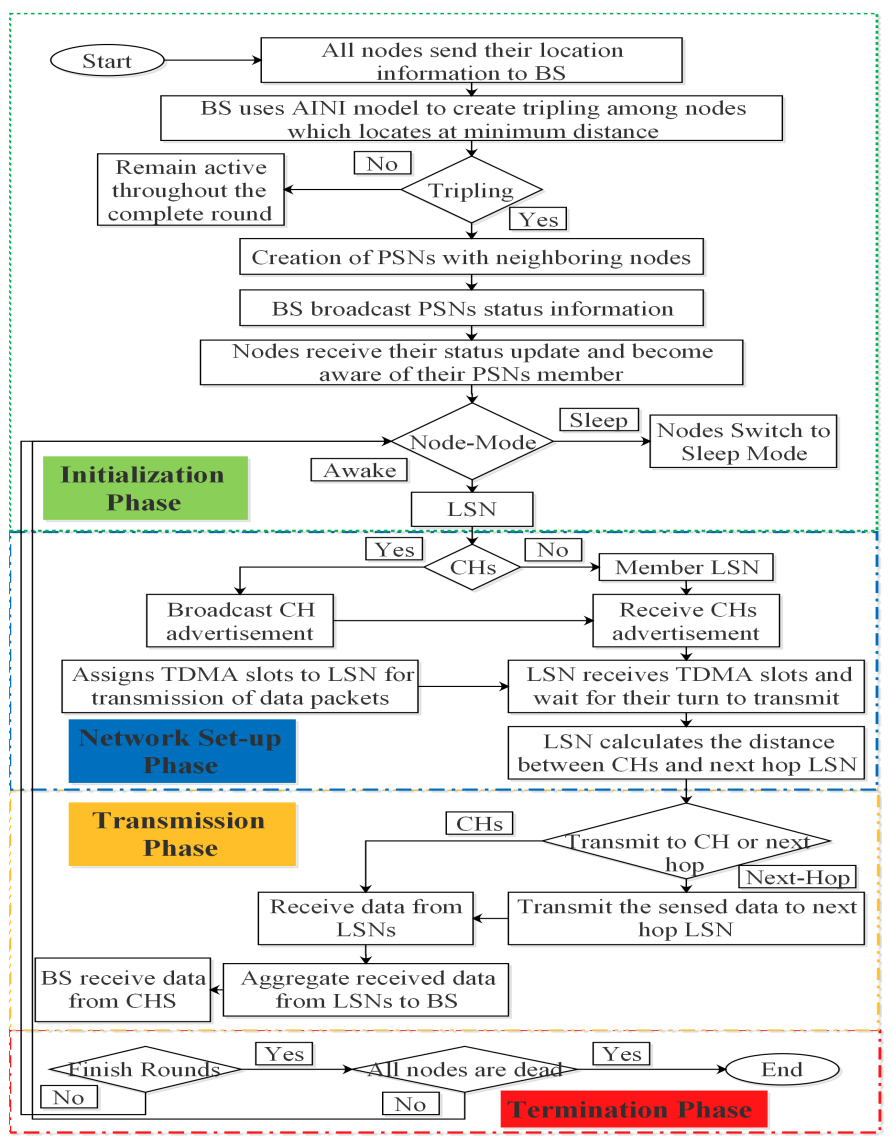

Fig. 3. Network Operations and Flowchart of AINI Routing Protocol

epoch. If the value of epoch is same for few low energy level nodes and high energy level nodes, they both have equal chances to become a $\mathrm{CH}$. Decision based on preferred ratio of being a $\mathrm{CHs}$ per round $\mathrm{CH}_{p r}$. Above mentioned protocols 
allows each node to become a $\mathrm{CH}$ after every $1 / \mathrm{CH}_{p r}$ round. As the nodes are consuming energy in every round, so the energy level cannot be the same after first round [27]. After first round $\mathrm{CH}$ selection of proposed protocol is based on residual energy of nodes. Active nodes for the first round will participate in $\mathrm{CH}$ selection process, and higher energy nodes will be selected as $\mathrm{CHs}$. In order to select $\mathrm{CHs}$, threshold is calculated with the following equation:

$$
T= \begin{cases}\frac{C H_{p r}}{1-C H_{p r} \times\left(r \times \frac{1}{C H_{p r}}\right) \times d} & \text { if } n \in \alpha \\ 0 & \text { otherwise }\end{cases}
$$

Where $\alpha$ shows the set of active-nodes in first round which are appropriate for $\mathrm{CH}, r$ is the round and $d$ is distance to BS . After calculating threshold, random number is generated at each node. If random number of node is $<$ the value of threshold, this node will be selected as a $\mathrm{CH}$. This $\mathrm{CH}$ broadcast announcement to whole network, only active-nodes will be able to receive this announcement. When a node chooses $\mathrm{CH}$ in its cluster range, this node will transmit a message using CSMA MAC protocol to evade collisions. This message contains the location and energy level of node. After receiving the message from nodes, $\mathrm{CH}$ will calculate the energy level and distance of each node to BS and choose the active nodes for next round and labelled them as $A N_{n r}$ in a table and broadcast this table to all active nodes in CCs. Furthermore, $\mathrm{CH}$ will assign TDMA slots to each active-node for communication and each node will transmit the sensed data in its assigned TDMA slot. Algorithm 1 shows the precise $\mathrm{CH}$ selection of proposed AINI protocol.

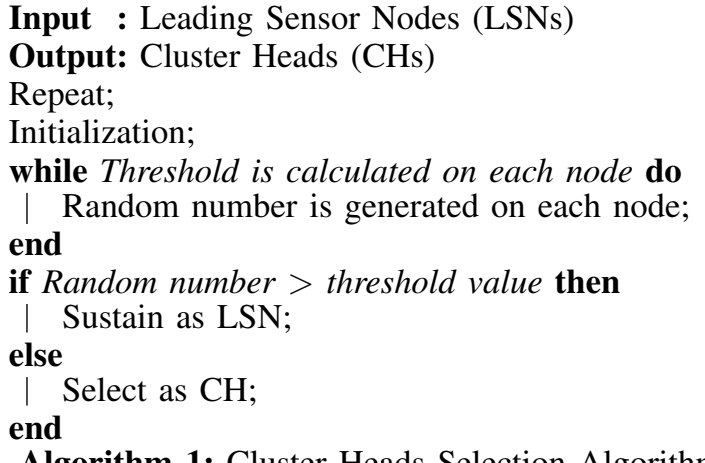

\section{B. Multi-Hop Routing}

Our proposed model develop unique two-layer multi-hop transmission phase in which both intra-cluster and inter-cluster communication adopt multi-hoping communication among sensor nodes to BS. The communication system utilizes first order radio model and furthermore nodes have capability to switch to multi-level transmission levels. If nodes have to participate in just intra-cluster communications then these nodes switch their transmission level to lowest one to save the energy resources which are being wasted during amplifications cost of transmissions. Similarly if nodes share the responsibilities of inter-cluster communications then these nodes switch to maximum transmission level to accommodate highest range of inter-cluster communications. Basic assumptions of first order radio model indicates the communications skills of sensor nodes which utmost can offer coverage of whole network region to small network area.

1) Intra-Cluster Multi-hop Routing: Each node in the network is able to determine its location, own distance from $\mathrm{CH}$ and neighbour node closer to $\mathrm{CH}$. If Node $m$ wants to transmit data packet, it will take the shortest path to transmit data to $\mathrm{CH}$ and creates the first route. Using the multi-hop routing, AINI protocol transmits routes with data in the overhead of packet, which improve the network lifetime and reduce the energy dissipation in hotspot area. Routes have adequate choice of pledge to fully disjoint multiple routes in condition of utilizing opportunistic routing. Trigonometric ratio and law of cosine is used to calculate the route width $R_{w}$ with transmitting range $r$ and distance $d$. By calculating the route width opportunistic routing can be utilized between the nodes in the range. Pseudocode of intra-cluster multi-hop routing is given in Algorithm 2. In order to transmit data through intra-cluster multi-hoping following number of hops are required:

$$
N_{h_{a}^{b}}=\left[\frac{a \times r_{\max }}{b \times r}\right]
$$

where, $N_{h_{a}^{b}}$ shows the require number hops to transmit data from source node $a$ towards the next-hop node $b$ and $r_{\max }$ is the maximum range of node.

2) Inter-Cluster Multi-Hop: For small networks intercluster multi-hop routing may not be useful because data travels among the $\mathrm{CHs}$ which often consume more energy than direct transmission. But for the large scale network with the large amount of sensor nodes requires more clusters and $\mathrm{CHs}$ in the network, these $\mathrm{CHs}$ transmit data to $\mathrm{BS}$ with significant amount of energy [28]. In order to minimize the energy consumption in long range transmission, we use intercluster multi-hop routing in our proposed protocol. Assuming that all $\mathrm{CHs}$ have the same range $R$ of transmission which helps $\mathrm{CHs}$ to communicate with each other. $\mathrm{CHs}$ located near to $\mathrm{BS}$ (their range covered by the $\mathrm{BS}$ ) have the number of hops $N_{h_{a}^{b}}=0$, while CHs locates away from BS calculate the distance of neighbouring $\mathrm{CHs}$ which is in the direction of BS. First hop of $\mathrm{CH}$ will be labelled as $N_{h^{b}}=1$ and the next hop will add its hop distance in $N_{h_{a}^{b}}$ and pass it to other neighbour $\mathrm{CH}$. Algorithm keeps choosing the hop distance until all $\mathrm{CHs}$ are connected to each other. After calculating the hop distance $\mathrm{CHs}$ will transmit data to the next hop $\mathrm{CH}$, next hop $\mathrm{CH}$ transmits this data with its own data to the next hop $\mathrm{CH}$, in this way data keep moving among $\mathrm{CH}$ until it reached to BS. Figure 4 and 5 shows the multi-hop routing with respect to rounds.

\section{Data Transmission}

To transmit data, proposed model adopts the lossless stepby-step multi-hop transmission [29]. In this transmission model , the transmission of $i$ bit message with node $n_{i}$ is executed successively. Such as; node transmit the received data with its own sensed data after arrival. $\delta$ denotes the non-transmitted data (or source data) packet of node $n_{i} ; \varepsilon\left(n_{i}, n_{j}\right)$ denotes as intermediate transmission result of node $n_{i}$ and node $n_{j} . \psi$ denotes the final transmission of node $n_{i}$ of all received data and its own data. When node $n_{i}$ receive data $\psi$ from node $n_{j}$ , node $n_{i}$ transmit $\psi_{j}$ with its own data (may be the source 
Input : Location of Source Node $\left(S_{n}\right)$, Number of Neighbour Nodes $\left(N_{n}\right)$, List of Neighbour, Distance from $S_{n}$ to distance $d$, Packet Transmission Ratio PTR, Transmission Range $\left(T_{R}\right)$

Output: Find the shortest route and deliver data to $\mathrm{CHs}$

Compute the number of total nodes;

Select $n$ number of nodes;

for each node $m$ do

Calculate the node range;

$R_{n}=d \times \sqrt{1-\left(2 d^{2}-r^{2} / 2 \times d^{2}\right)^{2}}$

$R_{w}=2 \times R_{n}$

Node $o$ is a neighbour of node $m$ with longest distance from $S_{n}$;

Node $m$ forward its sensed data to node $o$;

Random hop $R_{h}$ from $\left\{0, r_{\max } / r\right\}_{0}=0$;

while $R_{h_{0}}<R_{h}$ do

Farthest node from node $o$ is $o_{i}$ at leftmost(rightmost) routes and a distance $\mathrm{d}$ from BS is a same number of hops as node $o$; Node $o$ transmits packet to node $o_{i}$;

end

$R_{h_{o}}=R_{h_{o}}+1$, and $o=o_{i}$;

end

if node $m$ is closest to $\mathrm{CH}$ then

Transmit packet directly to $\mathrm{CH}$ and create major route;

Node $q$ is selected as random node $R_{n}$ from major route in non-hotspot area;

$N_{h_{a}^{b}}$ hops routed from node $q$ towards left route;

$N_{h_{a}^{b}}$ hops routed from node $q$ towards right route; else

Before convention of same-hop route, transmits data packets to $\mathrm{CH}$;

end

All data packets are reached at $\mathrm{CHs}$;

$\mathrm{CHs}$ aggregate all data packets to $\mathrm{BS}$;

Algorithm 2: Multi-Hoping Pseudo-code of AINI

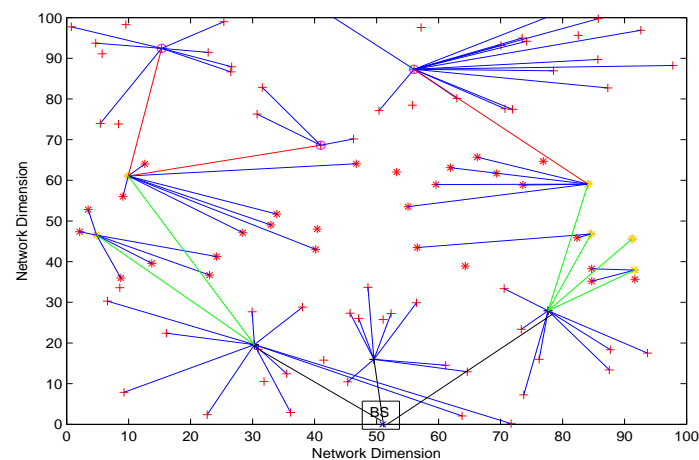

Fig. 4. Multi-hop Routing in Round (R)

data $\delta$ or intermediate data $\varepsilon$ ). If node $n_{i}$ transmitting data packet $\varepsilon$ and if data received from $n_{j}$ then $\psi_{j}=\varepsilon_{j}$, data to be transmitted are both source data, then the transmission formula will be:

$$
\varepsilon\left(n_{i}, n_{j}\right)=\max \left(\delta_{i}, \delta_{j}\right)+(1-c) \min \left(\delta_{i}, \delta_{j}\right)
$$

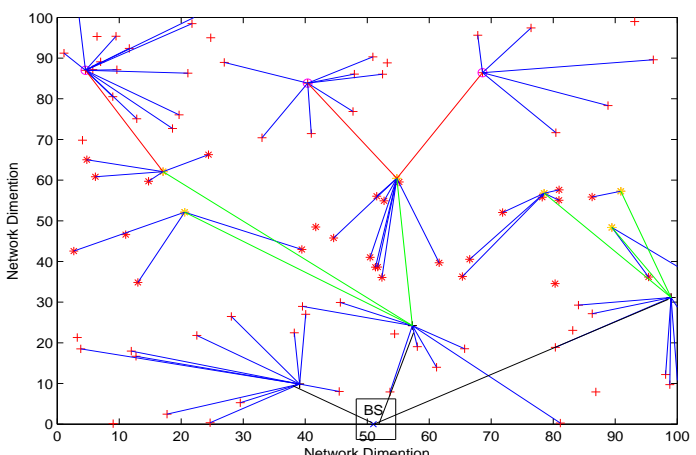

Fig. 5. Multi-hop Routing in Round ( $\mathrm{R}++)$

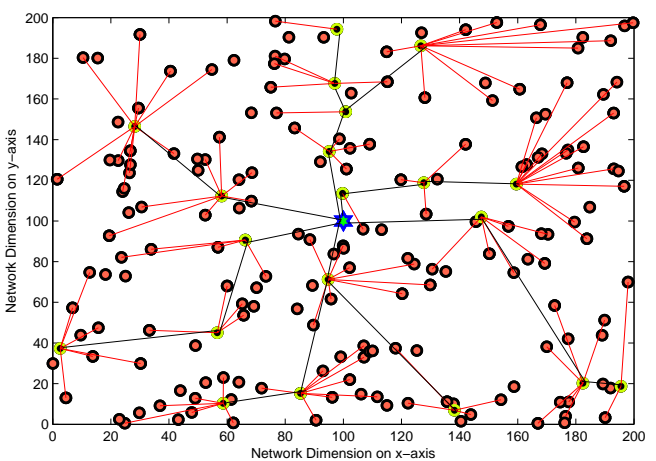

Fig. 6. Data Transmission between Intermediate Nodes

If transmitted data is not from source node, then intermediate transmission formula will be:

$$
\varepsilon\left(\varepsilon_{i}, \psi_{j}\right)=\max \left(\varepsilon_{i}, \psi_{j}\right)+\epsilon(1-c) \min \left(\varepsilon_{i}, \psi_{j}\right)
$$

$\epsilon$ is called forgetting factor. Its a decimal between ( 0 , $1)$, and $\varepsilon_{i}, \psi_{j}$ are the intermediate and final transmission of active nodes respectively. Figure 6 shows the data transmission between intermediate nodes.

\section{Energy Dissipation}

Active-mode nodes transmits the sensed data to $\mathrm{CH}$ using intra-cluster multi-hop routing in their allocated TDMA slots and sleep-mode nodes remain sleep until the next round. $\mathrm{CHs}$ received data from nodes and transmit it to BS. Depending on the distance between transceiver and receiver, we use the first order radio model and free space model in our proposed protocol [30]. We use this model for better comparison with existing protocols [17], [18], [19], [20], [21]. Parameter used by radio models are $E_{\text {elec }}=50 \mathrm{~nJ} / \mathrm{bit}, E_{f s}=$ $10 \mathrm{pJ} / \mathrm{bit} / \mathrm{m}^{2}$ and $E_{a m p}=0.0013 \mathrm{pJ} / \mathrm{bit} / \mathrm{m}^{4}$. To transmit a $i$ bit message to distance $d$, the radio expands:

$$
E_{T x}(i, d)= \begin{cases}i E_{\text {elec }}+E_{f s} i d^{2}, & \text { if } d<d o . \\ i E_{\text {elec }}+E_{a m p} i d^{4}, & \text { if } d \geq d o .\end{cases}
$$

Using the radio models, noticeable energy is saved in data transmission. If there are $N$ number of nodes in the network, and $N_{C H}$ is the best possible number of $\mathrm{CH}$, to calculate the 
average number of nodes in each cluster can be calculated by following equation:

$$
\left(\frac{N}{N_{C H}}-1\right)
$$

Transmission of Non-CH node, radio dissipates $E_{t x}$ and $E_{a m p}$ for transmitting to attain Signal-to-Noise ratio (SNR). Therefore, transmission of $i$ bit data packet by non- $\mathrm{CH}$ node will be:

$$
E_{n_{C H}}=\left(\frac{N}{N_{C H}}-1\right)\left(E_{t x} \times i \times E_{a m p} \times i \times d^{2}\right)
$$

$E_{n_{C H}}$ represents non-CH nodes, $E_{t x}$ and $E_{a m p}$ is transmitting energy and amplifier of nodes respectively, while $d$ is a distance of node towards BS. Receiving data by $\mathrm{CHs}$ from $N_{n_{C H}}$ nodes, the equation will be:

$$
E_{r c v}=\left(E_{r x} \times i\right)\left(\frac{N}{N_{C H}}-1\right)
$$

$E_{r c v}$ is the total received data at any $\mathrm{CH}$, and $E_{r x}$ is the total energy dissipated by $\mathrm{CH}$ while receiving this data. In order to aggregate this data towards BS, energy dissipates by $\mathrm{CH}$ will be:

$$
E_{T_{B S}}=\left(E_{d a t a} \times i\right)\left(\frac{N}{N_{C H}}\right)
$$

$E_{T_{B S}}$ is the transmission data towards BS, and $E_{d a t a}$ is the total data received by any $\mathrm{CH}$. In order to calculate the total dissipated energy by any $\mathrm{CH}$ to transmit this data, the equation will be:

$$
E_{T}=\left(E_{t x} \times i_{A D} \times E_{a m p} \times d^{2}\right)
$$

$E_{T}$ is the dissipated transmission energy of any $\mathrm{CH}$ to transmit the total data $i_{A D}$ received from all associated nodes. To calculate the total dissipated energy in one round, we use the following equation:

$$
E_{C H}=E_{T_{B S}}+E_{T}+E_{r c v}
$$

Total dissipated energy of $\mathrm{CHs}$ in one round is the sum of $E_{T_{B S}}$ data transmission towards BS, $E_{T}$ dissipated energy of $\mathrm{CH}$ while transmission and $E_{r c v}$ dissipated energy of $\mathrm{CH}$ while receiving.

\section{E. Node-Mode Decision}

As mentioned above, $\mathrm{CHs}$ generate a table of remaining energy and $D_{B S}$ of nodes and broadcast it to all CSNs. Nodes decides at the end of round weather to be in sleep-mode or in active-mode after reviewing this table. If a node is elected as active-node in next round $A N_{n r}$ it will turn $\mathrm{ON}$ its transceiver in the next round otherwise it will remain OFF until its get elected. CSNs nodes participating in tripling procedure turn
OFF their transceiver in consecutive two rounds when these nodes are not active as LSN node. Furthermore, their sleepmode continue until they elected again as LSN to start their active-mode. Some nodes face remote dispersion due to initial random deployment, so these nodes can miss the initial triplet bonding, thus these nodes remain in active-mode during the whole network operation. Algorithm 3 shows the node-mode decision whether to be in sleep-mode or in active-mode.

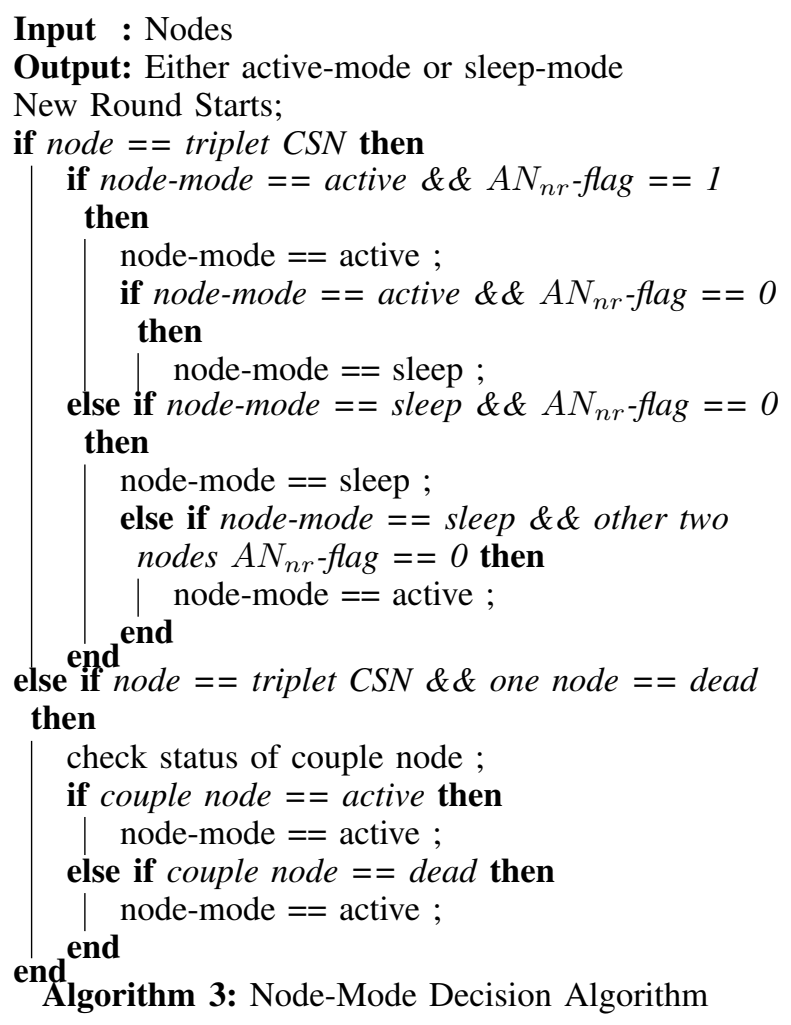

Initially nodes will check either they are selected for triplet CSNs or not, if node is selected for CSNs than it will check its flag in $A N_{n r}$ table. If flag is ON in $A N_{n r}$ table, this node will sustain as LSN. Similarly, all nodes check their status in the table, nodes which are in active-mode but their flag is OFF, they will turn OFF their transceiver and receiver and will remain in sleep-mode until their flag turn ON. CSNs will check the status of their triplet nodes in $A N_{n r}$. If node in sleep mode, it will check the flag of other two nodes, if their flags are OFF, it will turn itself into active-mode, if not it will remain OFF until next round. When a node in sleep mode, it will continuously check the status of their triplet CSNs. If a node in CSNs are in sleep mode or dead, this node will turn itself into active-mode. If one node is dead in triplet CSNs, then the decision will remain between coupled nodes. Both nodes check their flag status, one will be in active-mode and the other one in sleep-mode. If two nodes are dead in the triplet CSNs, last node will remain active throughout its lifetime. Nodes which are not selected for any triplet CSNs, will be in active mode throughout the network.

\section{EXPERIMENTS AND SimUlation RESUlts}

This paper introduces a neighbour-cooperation heterogeneity-aware traffic engineering for WSNs with 
the concept of tripling the sensor nodes. Following subsection explains the performance measures, methodology and simulation results.

\section{A. Performance Measures}

To analyze the performance of proposed protocol, we use the following metrics: leftmargin $=*$,labelsep $=5.8 \mathrm{~mm}$

- Network Stability (NS): Total time from start of network operation to the demise of first node.

- $\quad$ Network Instability (NI): Total time form demise of first node to the demise of last node.

- Network Lifetime (NL): Total time from start of network operation to the demise of last node.

- $\quad$ Energy Consumption Ratio (ECR): Total energy consume during the transmission and receiving of data packet.

- $\quad$ Death of First Node (DFN): Network operation from the start of network till the death of first node. It also measures the stability period of routing protocol.

- $\quad$ Death of Last Node (DLN): Network operation from the start of network till the death of last node, it helps to measure the instability period of routing protocol.

\section{B. Experiments Methodology}

To evaluate the performance of AINI, we have done simulations in MATLAB environment. Similar to other environments, such as OMNET ++ and ns-2 with the difference that MATLAB provides feature architecture and allows rapid simulations by combining multiple components. To provide the better presentation of experimental results we use OriginLab for deep analysis in few results. Multiple scenarios and various parameters are considered to evaluate the performance.

\section{Comparison with State-of-the-Art Algorithms}

The proposed AINI routing protocol is compared with state-of-the art routing protocols such as LEACH [17], LEACH-C [18], SEP [19], ESEP [20] and DEEC [21]. First we validate the proposed analysis model for AINI routing protocol then compare the proposed protocol by executing it in different network dimensions. To prove the performance of proposed protocol in large scale network, we took the simulations in two different scenarios. Specifically, simulation scenarios include 100 and 200 sensor nodes which are deployed in $100 \mathrm{~m} \times 100 \mathrm{~m}$ and $200 \mathrm{~m} \times 200 \mathrm{~m}$ network area with initial network energy $1 J, 1.5 J$ respectively, while the BS is located in the center of the network. To remove the error caused by randomness each simulation runs at least for five times and average is considered as a final result. Major simulation parameters are given in Table I.
TABLE I. Simulation Parameters

\begin{tabular}{lc}
\hline Sensor Nodes Scenario 1 & 100 \\
Sensor Nodes Scenario 2 & 200 \\
Network dimensions Scenario 1 & $100 \mathrm{~m} \times 100 \mathrm{~m}$ \\
Network dimensions Scenario 2 & $200 \mathrm{~m} \times 200 \mathrm{~m}$ \\
Heterogeneous Energy Level Scenario 1 & $1 \mathrm{~J}$ \\
Heterogeneous Energy Level Scenario 2 & $1.5 \mathrm{~J}$ \\
Required CHs Per Round & $10 \%$ \\
Transmission Energy Dissipation & $50 \mathrm{pJ} / \mathrm{bitj}$ \\
Data Packet Size & $4000 \mathrm{bit}$ \\
Transmission Energy & $50 \mathrm{~nJ} / \mathrm{bit}$ \\
Receiver Energy & $50 \mathrm{~nJ} / \mathrm{bit}$ \\
Amplifier Transmission Energy Dissipation & $100 \mathrm{pJ} / \mathrm{bit} / \mathrm{m2}$ \\
\hline
\end{tabular}

\section{Experiments}

It is noticed that, tripling of nodes extends the network lifetime and stability of network. Moreover, multi-hoping improves the lifetime by distributing the load of one $\mathrm{CH}$ among multiple $\mathrm{CHs}$. In the simulation results of first scenario, first node dies in proposed protocol after 2432 which shows the stability period is much better than LEACH, LEACH-C, SEP, ESEP and DEEC. Moreover, last node dies in proposed protocol at 5978 rounds, which shows the instability period of proposed protocol. It can be seen clearly in Figure 7 and 8 that proposed protocol produce better performance then state-of-the art routing protocols.

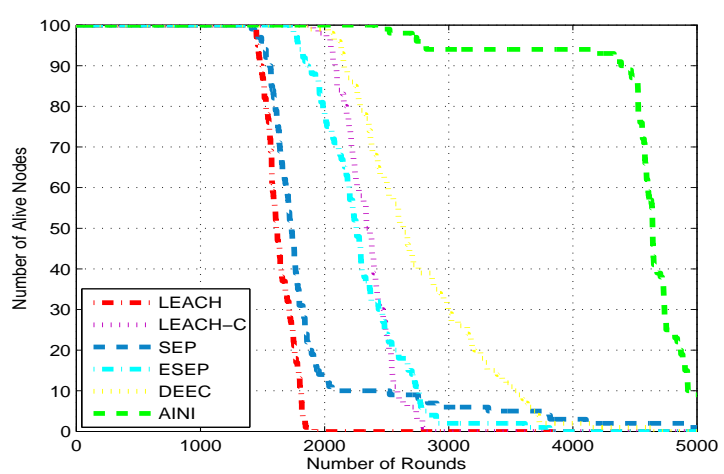

Fig. 7. Network Lifetime Respectively in Network of $100 \times 100$ with 100 Nodes

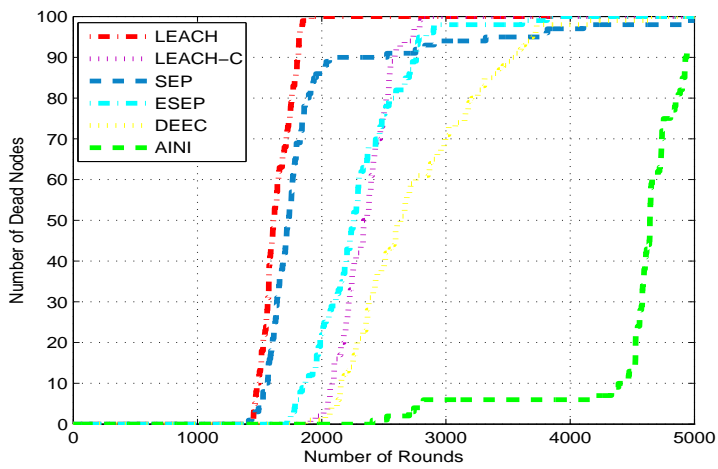

Fig. 8. Network Stability in Network of $100 \times 100$ with 100 Nodes

Figure 9 and 10 show the number of data packets transmitted towards $\mathrm{CH}$ and $\mathrm{BS}$ respectively. From these figures, it is examined that the multi-hop increases the number of sent packets to BS, because inter-cluster multi-hoping helps the $\mathrm{CHs}$ to transmit data to BS with minimum energy dissipation. Undoubtedly, proposed protocol improves the network lifetime 
and stability of network. Furthermore, it improves the throughput and reduces the energy dissipation throughout the network. This means, that proposed protocol is more energy efficient than previous well known existing protocols, because propose protocol allows the nodes to work with full functionality by improving the packet delivery ratio and reducing the delay in transmission.

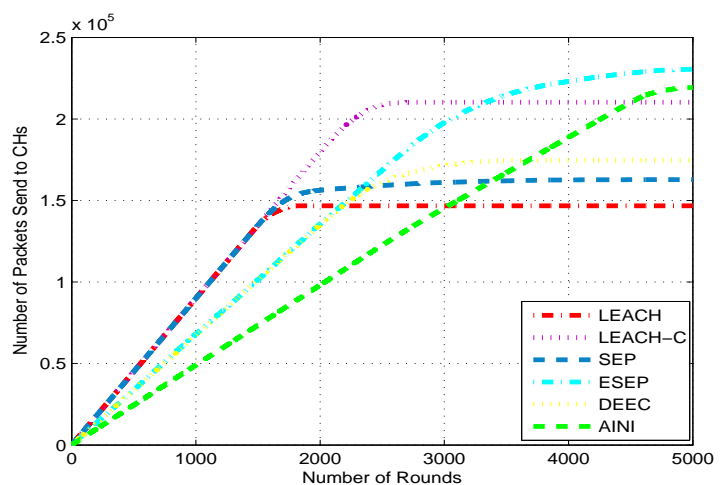

Fig. 9. Successfully Delivered Packets to CHs in Network of $100 \times 100$ with 100 Nodes

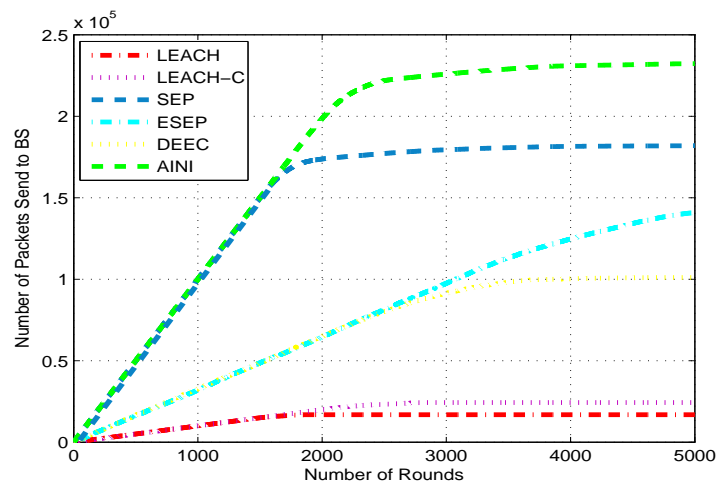

Fig. 10. Successfully Delivered Packets to BS in Network of $100 \times 100$ with 100 Nodes

Figure 11 and 12 shows the performance of proposed protocol for second scenario, in terms of network lifetime and network stability of proposed protocol with the comparison of other protocols. As per the results, our proposed protocol AINI outperforms in large scale heterogeneous network. The result presented in Figure 11 and 12 also demonstrate the instability period of proposed protocol at large scale which proves that AINI protocol have the minimum instability period.

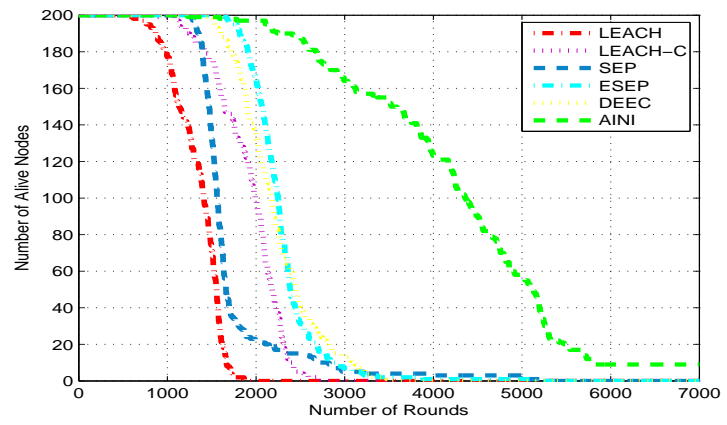

Fig. 11. Network Lifetime in Network of $200 \times 200$ with 200 Nodes

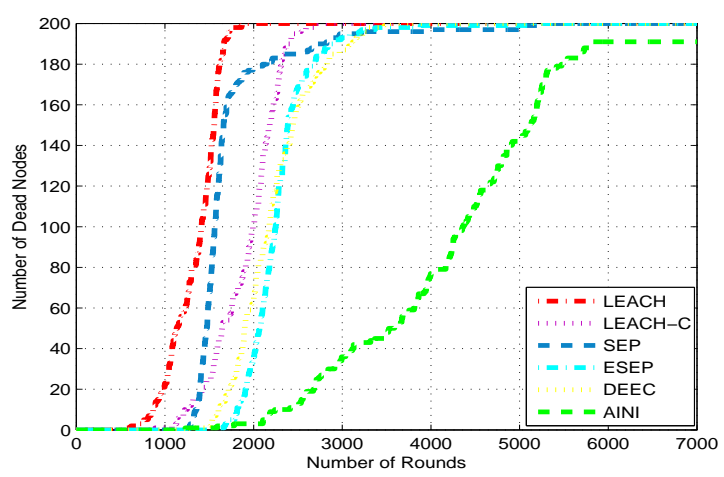

Fig. 12. Network Stability in Network of $200 \times 200$ with 200 Nodes

Figure 13 and 14 shows the comparison of overall network energy consumption with respect to rounds. It is noticed that proposed routing protocol is utilizing minimum energy in both graphs. First graph shows the network energy consumption of scenario 1 while second graph shows the energy consumption of scenario 2. It can be seen clearly in second graph that all other protocol are dead after 5500 rounds but proposed protocol is still surviving in terms of energy (joule).

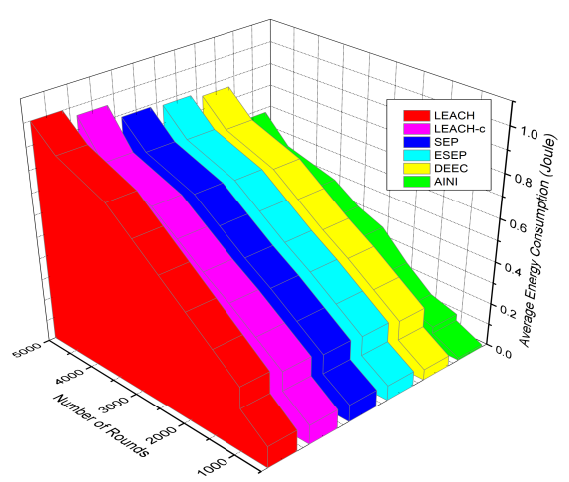

Fig. 13. Average Energy Consumption in Network of $100 m \times 100 m$

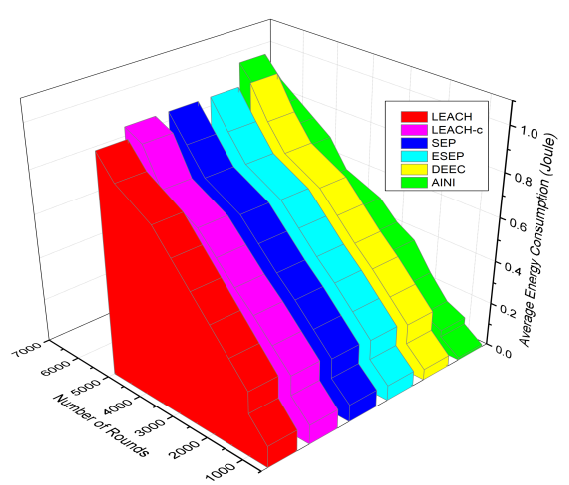

Fig. 14. Average Energy Consumption in Network of $200 m \times 200 m$

Figure 15 shows the comparative result of proposed protocol with other protocols in terms of heterogeneity-aware. Figure shows the impact of multiple heterogeneity where multiple network scenarios are considered and shows that proposed routing protocol obtains higher stability and longer network lifetime in larger network. 


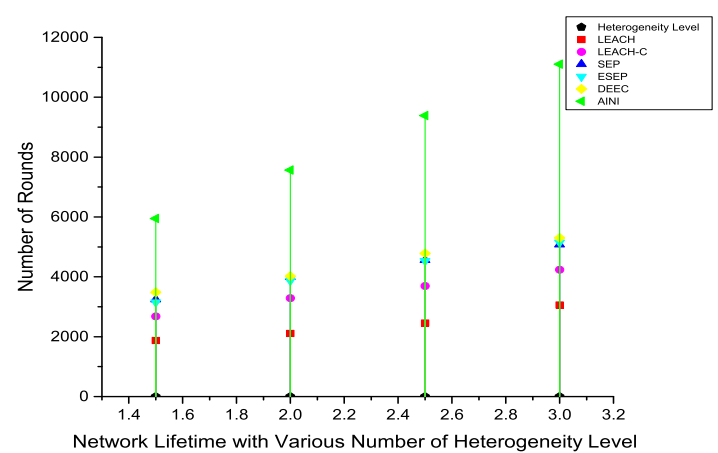

Fig. 15. Impact of Different Heterogeneity Level

\section{CONCLUSIONS AND FUtURE WORK}

This paper introduces a new technique NeighbourCooperation Heterogeneity-Aware Traffic Engineering for WSNs and proposed a new clustering routing protocol called Adaptive Inter-networking Improved routing protocol (AINI) to improve the stability, network lifetime, $\mathrm{CH}$ selection and average energy consumption. Furthermore, this protocol uses multi-hop (intra-cluster communication for transmission between nodes and $\mathrm{CHs}$ and inter-cluster communication for transmission between $\mathrm{CHs}$ and BS) routing which enhanced the reliability of network. Simulation results proved that proposed protocol is more energy efficient and perform far better than well known existing routing protocols such as, LEACH, LEACH-C, SEP, ESEP and DEEC. Mobility based AINI routing protocol is considered as future work.

\section{STATEMENT OF CONFLICT}

Authors of this paper: Christopher Mumpe, Da Tang, Muhammad Asad, Muhammad Aslam, Jing Chen, Jinsi Zhu, Luyuan Jin declares that there is no conflict of interest regarding the publication of this research article entitled "Neighbour-Cooperation Heterogeneity-Aware Traffic Engineering for Wireless Sensor Networks".

\section{NOTATIONS}

The following notations are used in this paper.

$\begin{array}{ll}C S N & \text { Child Sensor Node } \\ L S N & \text { Leading Sensor Node } \\ P S N & \text { Parent Sensor Node } \\ C H_{p r} & \text { Cluster Heads Per Round } \\ \alpha & \text { Set of Active-Nodes in First Round } \\ A N_{n r} & \text { Active-Nodes for Next Round } \\ R_{w} & \text { Route Width } \\ R_{n} & \text { Node Range } \\ R_{h} & \text { Random Hop } \\ S_{n} & \text { Source Node } \\ N_{h_{a}^{b}} & \text { Number Of Hops } \\ \delta & \text { Non-Transmitted Data } \\ \varepsilon & \text { Intermediate Transmission Result Between Two Nodes } \\ \psi & \text { Final Transmission } \\ \epsilon & \text { Forgetting Factor } \\ D_{B S} & \text { Distance to Base Station }\end{array}$

\section{REFERENCES}

[1] K. Sohraby, D. Minoli, and T. Znati, Wireless sensor networks: technology, protocols, and applications. John Wiley \& Sons, 2007.

[2] I. M. El Emary and S. Ramakrishnan, Wireless sensor networks: from theory to applications. CRC Press, 2013.

[3] S. Tyagi and N. Kumar, "A systematic review on clustering and routing techniques based upon leach protocol for wireless sensor networks," Journal of Network and Computer Applications, vol. 36, no. 2, pp. 623-645, 2013.

[4] Y. Peng, F. Al-Hazemi, R. Boutaba, F. Tong, I.-S. Hwang, and C.-H. Youn, "Enhancing energy efficiency via cooperative mimo in wireless sensor networks: State of the art and future research directions," IEEE Communications Magazine, vol. 55, no. 11, pp. 47-53, 2017.

[5] N. A. Pantazis, S. A. Nikolidakis, and D. D. Vergados, "Energyefficient routing protocols in wireless sensor networks: A survey," IEEE Communications surveys \& tutorials, vol. 15, no. 2, pp. 551-591, 2013.

[6] N. A. O. Al-Humidi and G. V. Chowdhary, "Comparative analysis of clustering algorithms for routing protocols in wireless sensor networks," 2017.

[7] M. I. Chidean, E. Morgado, E. del Arco, J. Ramiro-Bargueno, and A. J. Caamaño, "Scalable data-coupled clustering for large scale wsn," IEEE Transactions on Wireless Communications, vol. 14, no. 9, pp. 46814694, 2015.

[8] A. Fahad, N. Alshatri, Z. Tari, A. Alamri, I. Khalil, A. Y. Zomaya, S. Foufou, and A. Bouras, "A survey of clustering algorithms for big data: Taxonomy and empirical analysis," IEEE transactions on emerging topics in computing, vol. 2, no. 3, pp. 267-279, 2014.

[9] P. Neamatollahi, M. Naghibzadeh, S. Abrishami, and M.-H. Yaghmaee, "Distributed clustering-task scheduling for wireless sensor networks using dynamic hyper round policy," IEEE Transactions on Mobile Computing, vol. 17, no. 2, pp. 334-347, 2018.

[10] M. C. M. Thein and T. Thein, "An energy efficient cluster-head selection for wireless sensor networks," in Intelligent systems, modelling and simulation (ISMS), 2010 international conference on. IEEE, 2010, pp. 287-291.

[11] A. A. Abbasi and M. Younis, "A survey on clustering algorithms for wireless sensor networks," Computer communications, vol. 30, no. 1415, pp. 2826-2841, 2007.

[12] W.-P. Chen, J. C. Hou, and L. Sha, "Dynamic clustering for acoustic target tracking in wireless sensor networks," IEEE transactions on mobile computing, vol. 3, no. 3, pp. 258-271, 2004.

[13] A. Wang, D. Yang, and D. Sun, "A clustering algorithm based on energy information and cluster heads expectation for wireless sensor networks," Computers \& Electrical Engineering, vol. 38, no. 3, pp. 662-671, 2012.

[14] M. Pramanick, P. Basak, C. Chowdhury, and S. Neogy, "Analysis of energy efficient wireless sensor networks routing schemes," in Emerging Applications of Information Technology (EAIT), 2014 Fourth International Conference of. IEEE, 2014, pp. 379-384.

[15] B. Guo and Z. Li, "A dynamic-clustering reactive routing algorithm for wireless sensor networks," Wireless Networks, vol. 15, no. 4, pp. 423-430, 2009.

[16] J.-J. Liaw, C.-Y. Dai, and Y.-J. Wang, "The steady clustering scheme for heterogeneous wireless sensor networks," in Ubiquitous, Autonomic and Trusted Computing, 2009. UIC-ATC'09. Symposia and Workshops on. IEEE, 2009, pp. 336-341.

[17] W. R. Heinzelman, A. Chandrakasan, and H. Balakrishnan, "Energyefficient communication protocol for wireless microsensor networks," in System sciences, 2000. Proceedings of the 33rd annual Hawaii international conference on. IEEE, 2000, pp. 10-pp.

[18] W. B. Heinzelman, A. P. Chandrakasan, and H. Balakrishnan, "An application-specific protocol architecture for wireless microsensor networks," IEEE Transactions on wireless communications, vol. 1, no. 4, pp. 660-670, 2002.

[19] G. Smaragdakis, I. Matta, and A. Bestavros, "Sep: A stable election protocol for clustered heterogeneous wireless sensor networks," Boston University Computer Science Department, Tech. Rep., 2004.

[20] F. A. Aderohunmu, J. D. Deng et al., "An enhanced stable election protocol (sep) for clustered heterogeneous wsn," Department of Information Science, University of Otago, New Zealand, 2009. 
[21] L. Qing, Q. Zhu, and M. Wang, "Design of a distributed energy-efficient clustering algorithm for heterogeneous wireless sensor networks," Computer communications, vol. 29, no. 12, pp. 2230-2237, 2006.

[22] A. Roshini and H. Anandakumar, "Hierarchical cost effective leach for heterogeneous wireless sensor networks," in Advanced Computing and Communication Systems, 2015 International Conference on. IEEE, 2015, pp. 1-7.

[23] F. Xiangning and S. Yulin, "Improvement on leach protocol of wireless sensor network," in Sensor Technologies and Applications, 2007. SensorComm 2007. International Conference on. IEEE, 2007, pp. 260-264.

[24] A. Manjeshwar and D. P. Agrawal, "Teen: a routing protocol for enhanced efficiency in wireless sensor networks," in null. IEEE, 2001, p. 30189a.

[25] A. Garg et al., "Distance adaptive threshold sensitive energy efficient sensor network (dapteen) protocol in wsn," in Signal Processing, Computing and Control (ISPCC), 2015 International Conference on. IEEE, 2015, pp. 114-119.
[26] A. Manjeshwar and D. P. Agrawal, "Apteen: A hybrid protocol for efficient routing and comprehensive information retrieval in wireless sensor networks," in ipdps. IEEE, 2002, p. 0195b.

[27] V. Gupta and R. Pandey, "An improved energy aware distributed unequal clustering protocol for heterogeneous wireless sensor networks," Engineering Science and Technology, an International Journal, vol. 19, no. 2, pp. 1050-1058, 2016.

[28] S. Khurana and K. R. Rekha, "Energy efficient of inter cluster multihop routing protocol for wireless sensor network," 2016.

[29] S. Chowdhuri, S. Chakraborty, N. Dey, S. S. Chaudhuri, and P. Banerjee, "Propagation analysis of mimo ad hoc network in hybrid propagation model and implement less propagation loss algorithm to find the minimum loss route," International Journal of Information and Communication Technology, vol. 10, no. 1, pp. 66-80, 2017.

[30] J. Banerjee, S. K. Mitra, and M. K. Naskar, "Comparative study of radio models for data gathering in wireless sensor network," International Journal of Computer Applications, vol. 27, no. 4, 2011. 